\title{
Numerical study of the square cup stamping process: a stochastic analysis
}

André Filipe Gomes Pereira, Miguel Fernandes Ruivo, Marta Cristina Oliveira, José Valdemar Fernandes and Pedro

$$
\text { André Prates }
$$

André Filipe Gomes Pereira. University of Coimbra, Centre for Mechanical Engineering, Materials and Processes (CEMMPRE), Department of Mechanical Engineering, Polo II - Pinhal de Marrocos, 3030-788 Coimbra, Portugal Corresponding author: andre.pereira@dem.uc.pt

Miguel Fernandes Ruivo. University of Coimbra, Centre for Mechanical Engineering, Materials and Processes (CEMMPRE), Department of Mechanical Engineering, Polo II - Pinhal de Marrocos, 3030-788 Coimbra, Portugal

Marta Cristina Oliveira. University of Coimbra, Centre for Mechanical Engineering, Materials and Processes (CEMMPRE), Department of Mechanical Engineering, Polo II - Pinhal de Marrocos, 3030-788 Coimbra, Portugal

José Valdemar Fernandes. University of Coimbra, Centre for Mechanical Engineering, Materials and Processes (CEMMPRE), Department of Mechanical Engineering, Polo II - Pinhal de Marrocos, 3030-788 Coimbra, Portugal

Pedro André Prates. University of Coimbra, Centre for Mechanical Engineering, Materials and Processes (CEMMPRE),

Department of Mechanical Engineering, Polo II - Pinhal de Marrocos, 3030-788 Coimbra, Portugal

Department of Mechanical Engineering, Centre for Mechanical Technology \& Automation (TEMA), University of Aveiro, 3810-193 Aveiro, Portugal

Abstract. The industrial demand for products with better quality and lower production costs have encouraged the widespread application of the finite element analysis (FEA) in the development and optimization of sheet metal forming processes. To ensure that the FEA solutions are reliable and robust it is important to take into account the uncertainties that inevitably arise in a real industrial environment. In this context, a numerical study on the influence of the material and process uncertainty in the stamping results of a square cup is presented. In this analysis, it is assumed uncertainty in the elasticity properties, hardening law parameters, anisotropy coefficients, blank thickness, friction coefficient and in the blank holder force. The effect of the uncertainty in these input parameters is evaluated in the punch force, equivalent plastic strain, thickness and cup geometry. Firstly, quasi-Monte Carlo method was used to evaluate the variability in the simulation outputs, considering the uncertainty of the input parameters. This analysis shows that the geometry is the output most sensitive to the uncertainty of the input parameters. Afterwards, a variance-based sensitivity analysis was carried out to identify the input parameters that most influence the output variability. It was concluded that the hardening law parameters and the anisotropy coefficients have the most influence in the stamping results variability of a square cup.

Keywords. Uncertainty Quantification, Sensitivity Analysis, Sobol’s Indices, Sheet Metal Forming Processes, Square Cup

\section{Introduction}

Sheet metal forming processes are among the most common and important metal working operations associated with the automotive, aeronautics and metalworking industries. Finite Element Analysis (FEA) is a well-established tool to predict the deformation processes and the factors limiting the formability of the components, and thus reducing the time and costs associated with the development and optimization of sheet metal forming processes. The traditional application of FEA is based on a deterministic approach that does not take into account the uncertainty that inevitably arise in a real industrial environment. Col [1] indicated that these uncertainties can be related with the variability in the material behaviour, process conditions, forming tools, lubrication and other random sources. Li [2] stated that these uncertainties have a significant influence in the quality of the formed component, leading to unreliable and non-robust solutions, and to the eventual rejection of the component. For these reasons, the stochastic modelling and uncertainties quantification of sheet metal forming processes are of current industrial interest. 
Numerical study of the square cup stamping process: a stochastic analysis

In recent years, several researchers have modelled the influence of the uncertainty sources on the final product variability, by resorting to Monte Carlo method [3, 4], design of experiences techniques [5, 6] and metamodels [7, 8]. The application of any of these methods requires the run of several numerical simulations of the forming process with distinct input values, in order to evaluate the variability of the forming results. Sensitivity analysis is generally coupled with these methods to quantify the influence of each source of uncertainty in the variability of the forming results. In sheet metal forming processes, different types of sensitivity analyses have been applied, such as variance-based methods [9], regression [10], derivative-based methods [11] and methods based on experimental designs [6].

In this work, a quasi-Monte Carlo method and a variance-based sensitivity analysis (Sobol's indices [12]), will be used to numerically evaluate the influence of the uncertainties associated with 11 input parameters (elasticity properties, hardening law parameters, anisotropy coefficients, blank thickness, friction coefficient and the blank holder force) in the stamping results variability of a square cup [13]. The effect of the uncertainty in these input parameters is evaluated in the punch force, equivalent plastic strain, thickness reduction and geometry of the cup. The stochastic analysis will allow to rank the influence of each uncertainty source in the forming results and to identify the regions of the square cup more affected by the inputs uncertainty. With this knowledge it is expected to identify the uncertainty factors that most affect the results in different regions of the cup, and that are essential to control in order to guarantee product conformity with the intended requirements. Additionally, this work intends to fill the lack of research studies associated with the variability of the square cup benchmark, which is one of the most used test cases [7, 14-17].

\section{Stochastic Model}

\subsection{Numerical Model}

The numerical model of the Square Cup forming process is shown in Figure 1. The geometry of the tools was based on the benchmark of the conference NUMISHEET' 93 [13]. In this process, a constant blank holder force (BHF) is applied to control the material flow. Then, the punch is moved until a total displacement of $40 \mathrm{~mm}$ is reached. After that, the tools are removed, promoting the springback of the square cup. Due to material and geometric symmetries, and to reduce the computational cost, only a quarter of the model is simulated. The square blank has an initial thickness $t_{0}$, and a side length of $75 \mathrm{~mm}$. The blank is discretized with a total of 1800 (8-node hexahedral solid) elements, with 2 elements in thickness and 30 elements in each side. The contact between the blank and the tools is described by the Coulomb's law with a constant friction coefficient, $\mu_{0}$. The tools were modelled using Nagata patches [18]. The simulations were carried out with the in-house code DD3IMP (Deep Drawing 3D Implicit Code) [19]. All the numerical simulations were performed on computers equipped with an Intel ${ }^{\circledR}$ Core $^{\mathrm{TM}}$ i7-8700K Hexa-Core processor $(4.7 \mathrm{GHz})$. The average computational time of each numerical simulation was 4 minutes and 34 seconds. 

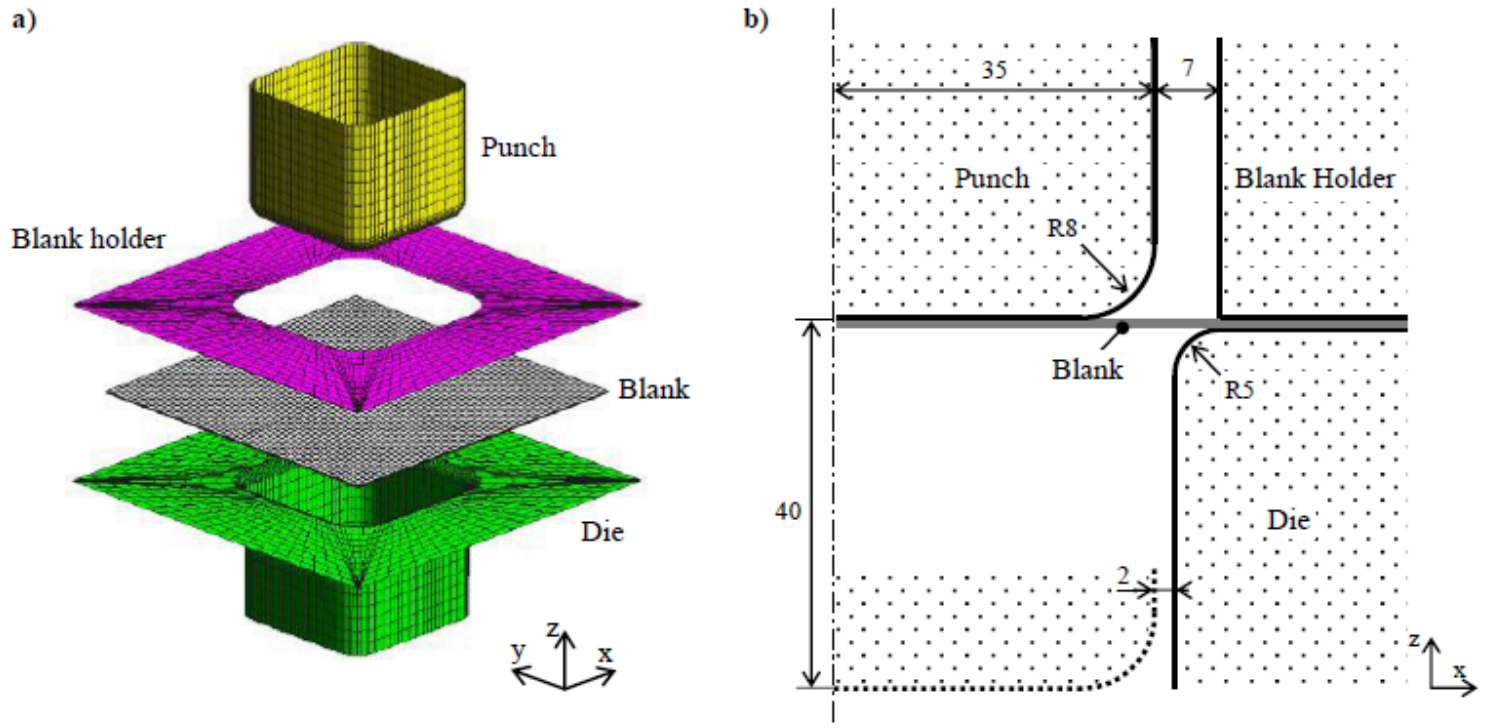

Fig. 1. Square cup forming process (a) Numerical model [20]; (b) Dimensions of the tools in mm

The constitutive model of the sheet assumes that: (i) the elastic behaviour is isotropic and is defined by the generalized Hooke's law, where the Young's modulus, E, and the Poisson's ratio, v, are elasticity parameters; (ii) the plastic behaviour is described by the orthotropic Hill'48 yield criterion and the Swift isotropic hardening law. The Hill'48 yield criterion is defined by the following equation:

$$
F\left(\sigma_{y y}-\sigma_{z z}\right)^{2}+G\left(\sigma_{z z}-\sigma_{x x}\right)^{2}+H\left(\sigma_{x x}-\sigma_{y y}\right)^{2}+2 L \tau_{y z}^{2}+2 M \tau_{x z}^{2}+2 N \tau_{x y}^{2}=Y^{2}
$$

where $\sigma_{x x}, \sigma_{y y}, \sigma_{z z}, \tau_{x y}, \tau_{y z}$ and $\tau_{x z}$ are the components of the Cauchy stress tensor defined in the orthotropic coordinate system of the material (Oxyz); F, G, H, L, M and $N$ are anisotropy parameters and $Y$ is the yield stress. The parameters of the Hill' 48 criterion follow the condition $G+H=1$, and so the yield stress, $Y$, is comparable to the uniaxial tensile stress along the rolling direction of the sheet. The parameters $L$ and $M$ were set to 1.5 , as in isotropy (von Mises). The parameters $F, G, H$ and $N$ can be obtained from the anisotropy coefficients $r_{0}, r_{45}$ and $r_{90}$, through the following relations:

$$
F=\frac{r_{0}}{r_{90}\left(r_{0}+1\right)}, G=\frac{1}{r_{0}+1}, H=\frac{r_{0}}{r_{0}+1} \text { and } N=\frac{1}{2} \frac{\left(r_{0}+r_{90}\right)\left(2 r_{45}+1\right)}{r_{90}\left(r_{0}+1\right)}
$$

The yield stress evolution during deformation, $Y=Y\left(\bar{\varepsilon}^{p}\right)$, is described by the Swift hardening law:

$$
Y=C\left(\varepsilon_{0}+\bar{\varepsilon}^{p}\right)^{n}
$$




\subsection{Input and Output Parameters}

A DC06 steel sheet is used in this work, the values of the material coefficients, namely $E, v, n, C, Y_{0}, r_{0}, r_{45}$ and $r_{90}$, are assumed to follow a normal distribution with mean, $\mu$, and standard deviation, $\sigma$, whose values are indicated in Table 1 [21]. Besides that, it is also assumed uncertainty in the blank thickness, $t_{0}$, friction coefficient, $\mu_{0}$, and blank holder force, $B H F$. These values are also assumed to follow a normal distribution with mean and standard deviation values given in Table 1 [21].

Table 1. Mean and standard deviation values that characterizes the normal distribution of each input parameter [21]

\begin{tabular}{lllllllllllll}
\hline & $\mathrm{E}[\mathrm{GPa}]$ & $v$ & $n$ & $C[\mathrm{MPa}]$ & $Y_{0}[\mathrm{MPa}]$ & $r_{0}$ & $r_{45}$ & $r_{90}$ & $t_{0}[\mathrm{~mm}]$ & $\mu_{0}$ & $\mathrm{BHF}[\mathrm{N}]$ \\
\hline$\mu$ & 206.00 & 0.300 & 0.259 & 565.32 & 157.12 & 1.790 & 1.510 & 2.270 & 0.780 & 0.1440 & 2450.0 \\
\hline$\sigma$ & & & & & & & & & & & & \\
\hline
\end{tabular}

The influence of the input parameters uncertainty was evaluated for four numerical results, the punch force $(P F)$, the equivalent plastic strain $\left(\bar{\varepsilon}^{p}\right)$, the thickness change $(T C)$ and the geometry change $(G C)$. The $P F$ and the $\bar{\varepsilon}^{p}$ values are directly given by the simulation software. The $T C$ and the $G C$ are defined as follows:

$$
\begin{gathered}
T C[\%]=\frac{t_{0}-t_{f}}{t_{0}} \times 100 \\
G C[\mathrm{~mm}]=\sqrt{\left(\bar{x}_{f}-x_{f}\right)^{2}+\left(\bar{y}_{f}-y_{f}\right)^{2}+\left(\bar{z}_{f}-z_{f}\right)^{2}}
\end{gathered}
$$

Where $t_{0}$ is the initial thickness value, $t_{f}$ is the final thickness value of a given cross section; $\left(\bar{x}_{f}, \bar{y}_{f}, \bar{z}_{f}\right)$ is the final position of a given node for the numerical simulation with the mean values of the input parameters (see Table 1), and $\left(x_{f}, y_{f}, z_{f}\right)$ is the final position of the same node but for the numerical simulations with uncertainty in the input parameters. In this way, $G C$ quantifies the positional difference of a given node between the deterministic simulation (i.e., with the mean input values) and the simulations with uncertainty in the input parameters. $\bar{\varepsilon}^{p}, T C$ and $G C$ are assessed for all the nodes of the simulation in order to evaluate the variability on these outputs for the different regions of the square cup. The $P F$ values are assessed for several displacements of the punch, up to the final punch displacement of $40 \mathrm{~mm}$.

\subsection{Variability in the forming results}

A quasi-Monte Carlo method was used to evaluate the variability of the forming results. This method was coupled with Sobol sequence to generate the sample of input parameters [22]. This sequence allows a faster convergence when compared to random sampling [23]; and the possibility of easily increase the sample size [24], if necessary. A sample size of 3000 simulations of the square cup was chosen. This value guarantees the convergence of the output statistical measures used in this work (mean and standard deviation of the forming results).

The mean and standard deviation of the $P F$ values along the punch displacement are shown in Figure 2. Figure 3 
presents the mean and standard deviation values of the forming results, $\bar{\varepsilon}^{p}, T C$ and $G C$, along the square cup. Both figures show that the mean and standard deviation results have similar distributions/trends. In fact, the critical regions, with large mean values of equivalent plastic strain, thickness reduction and geometry change, are also the regions that are more affected by the uncertainty in the input parameters. In this sense, the input parameters uncertainty can be a significant factor in the success of the forming process. The coefficient of variation (i.e., $\sigma / \mu$ ) for the points were the mean values of $P F, \bar{\varepsilon}^{p}, T C$ and $G C$ are maximum, are equal to 5.02\%, 3.12\%, 4.87\% and 56.75\%, respectively. Based on these coefficients of variation, it can be concluded that the $G C$ is the result more affected by the uncertainty in the input parameters. Figure 3 shows that the flange and wall of the square cup are the regions most influenced by the uncertainty in the input parameters. In contrast, the bottom of the cup is the region less influenced by the uncertainty in the input parameters, probably due to the small deformations and springback that occur in this region.
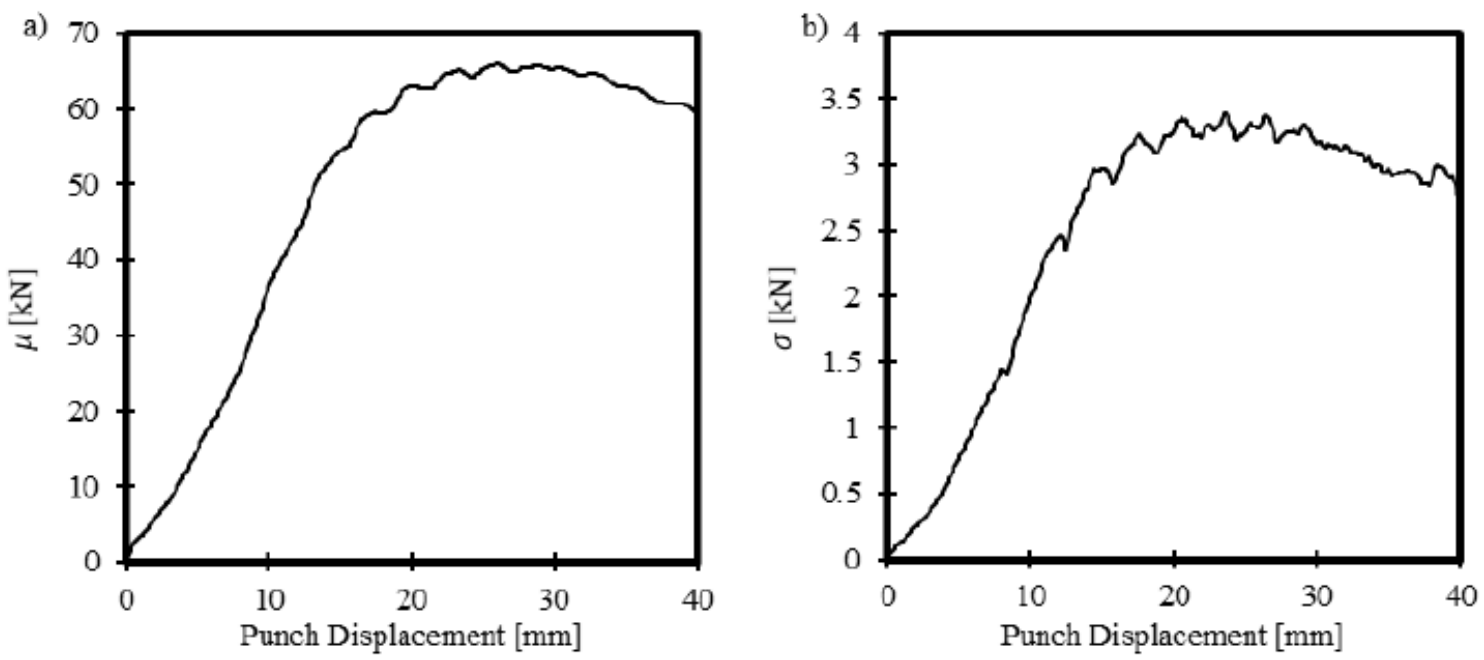

Fig. 2. Evolution of the: a) mean and b) standard deviation values of the punch force $(P F)$, along the punch displacement 

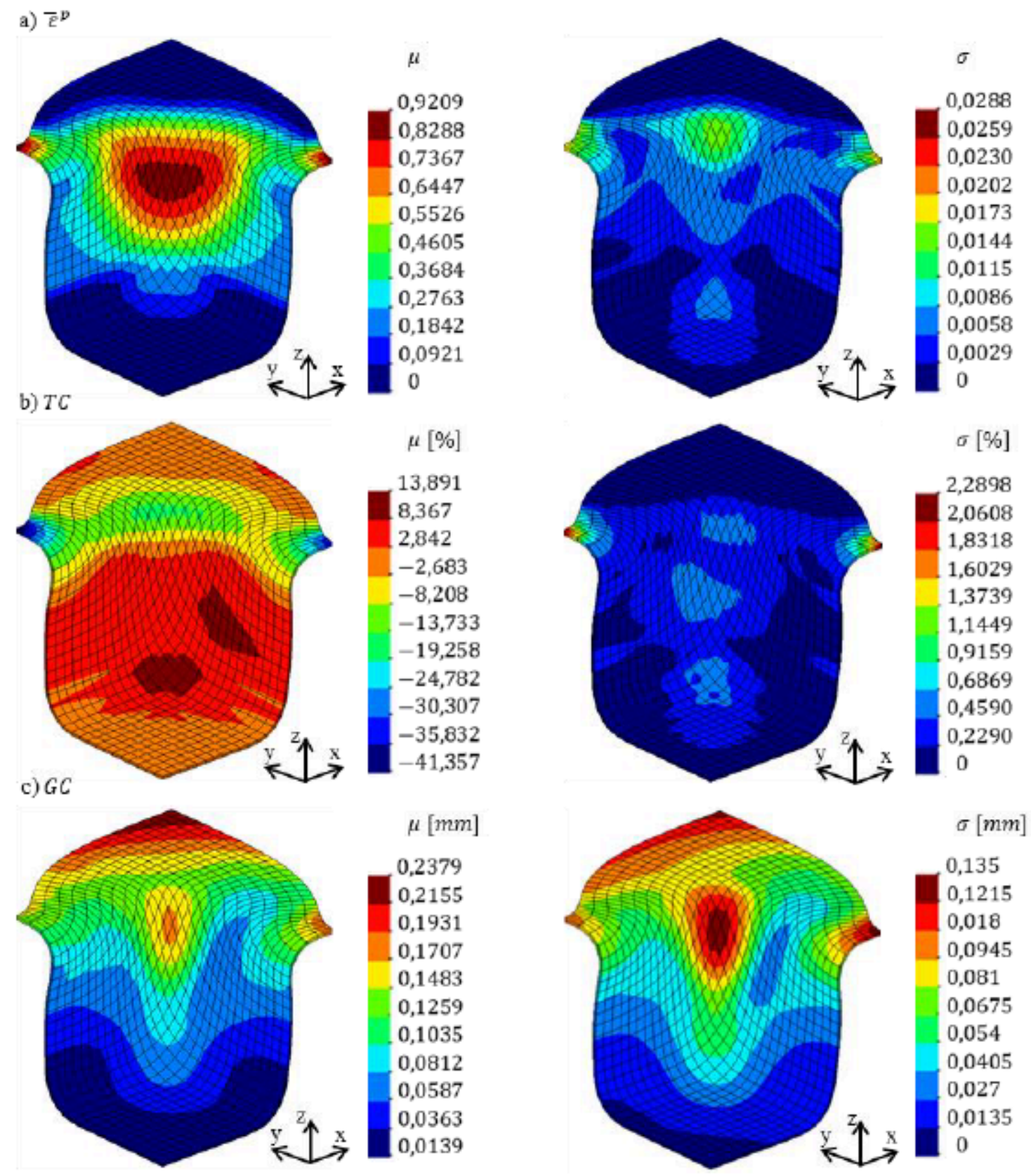

Fig. 3. Mean and standard deviation of the forming results: a) $\bar{\varepsilon}^{p}$, b) $T C$ and c) $G C$

\section{Sensitivity analysis}

\subsection{Sobol's Indices}

In order to evaluate the input parameters that most influence the forming results, a variance-base sensitivity analysis is carried out in this section. In specific, Sobol's indices will be used to quantify this influence. These indices are defined as follows [12]: 


$$
S_{i}=\frac{V\left[E\left(U \mid X_{i}\right)\right]}{V(U)}
$$

where $V(U)$ is the unconditional variance of the result $U$, and $V\left[E\left(U \mid X_{i}\right)\right]$ is the conditional variance of the expected value of $U$ when all input parameters, but $X_{i}$, are fixed. This equation defines the $1^{\text {st }}$ order index, $S_{i}$, which quantifies the individual influence of each input parameter, $X_{i}$, on the result $U$. In addition to $1^{\text {st }}$ order indices, it can also be defined total sensitivity indices, $S_{i}{ }^{T}$, as follows [12]:

$$
S_{i}^{T}=1-\frac{V\left[E\left(U \mid X_{\sim}\right)\right]}{V(U)}
$$

where $V\left[E\left(U \mid X_{\sim}\right)\right]$ is the conditional variance of the expected value of $U$ when the input parameter $X_{i}$ is fixed. The total sensitivity indices, $S_{i}{ }^{T}$, quantify not only the individual influence of each input parameter, $X_{i}$, on the result $U$, but also the influence of the interactions between the input parameter $X_{i}$ and the remaining, on the result $U$. The influence of the interactions between input parameters, int, is evaluated by:

$$
S_{i n t}=1-\sum_{i=1}^{k} S_{i}
$$

where $k$ is the total number of input parameters.

The indices $S_{i}$ and $S_{i}{ }^{T}$ are numerically evaluated, according to the method proposed in [25]. To improve the stabilization of the indices, by this method, the estimators proposed in [26] were used. A base number of 3000 simulations was used in this work, which implies 36000 additional simulations to evaluate the sensitivity indices for the 11 input parameters. The stabilization of the indices values occurs for a base number of approximately 1000 simulations.

\subsection{Sensitivity for the maximum values of the forming results}

This section presents the main results of the sensitivity analysis for the maximum values of the forming results, $P F_{\max }$, $\bar{\varepsilon}^{p} \max , T C_{\max }$ and $G C_{\max }$. For each of these results, the 1 st order indices, $S_{i}$, for the 11 input parameters and the index, $S_{\text {int }}$, associated with the interactions between these parameters, were evaluated. Figure 4 shows these indices for the maximum values of the forming results. It can be concluded from this figure that:

- the uncertainty in the hardening parameters, $C$ and $n$, and the blank thickness $t_{0}$, is responsible for over $90 \%$ of the variability in the $P F_{\max }$ values;

- the uncertainty in the hardening parameter $n$, and the anisotropy coefficients, $r_{90}$ and $r 45$, is responsible for about $90 \%$ of the variability in the $\bar{\varepsilon}^{p} \max$ and $T C_{\max }$ values;

- the uncertainty in the anisotropy coefficient $r_{90}$ and in other input parameters with strong interactions is responsible for about $80 \%$ of the variability in the $G C_{\max }$ values;

- the uncertainty in the input parameters, $E, v, Y_{0}, \mu_{0}$ and $B H F$ has a small individual influence in the results, although the interactions between these and other parameters can show a significant effect in the variability 
Numerical study of the square cup stamping process: a stochastic analysis

of the $G C_{\max }$ values. The study of each of these interactions will not be part of this work as it would require a huge computational cost.

\subsection{Maximum Sensitivity Indices per region}

This section presents an evaluation of the input parameter that most influences the forming results $\bar{\varepsilon}^{p}, T C$ and $G C$, in each region of the square cup. Figure 5 shows the input parameters whose 1 st order index is maximum for each node of the square cup, on the forming results $\bar{\varepsilon}^{p}, T C$ and $G C$. From this figure it can be concluded that:

- At the bottom of the square cup, the uncertainty of the hardening parameter $n$ is the main responsible for the variability in the $\bar{\varepsilon}^{p}, T C$ and $G C$ values. Nevertheless, the variability of these forming results is small at this region (see Figure 3);

- At the wall of the square cup, the uncertainty of the hardening parameter $n$ and the anisotropy coefficient $r 90$, is the main responsible for the variability in $\bar{\varepsilon}^{p}, T C$ and $G C$. The results of $\bar{\varepsilon}^{p}$ and $T C$ are also affected by the uncertainty in the hardening parameter $C$, while the uncertainty of the anisotropy coefficient $r_{0}$ influences the results of $T C$ and $G C$;

- At the flange of the square cup, the uncertainty of the anisotropy coefficients $r_{0}$ and $r_{90}$ is responsible for the major variability in the $\bar{\varepsilon}^{p}, T C$ and $G C$ values. The uncertainty of the hardening parameters $n$ and $C$ also affects the variability of $\bar{\varepsilon}^{p}$ and $T C$, but in a region where the variability of these results is small (see Figure 3). The variability of $G C$ is also affected, to a lesser extent, by the uncertainty of the anisotropy coefficient $r_{45}$. 

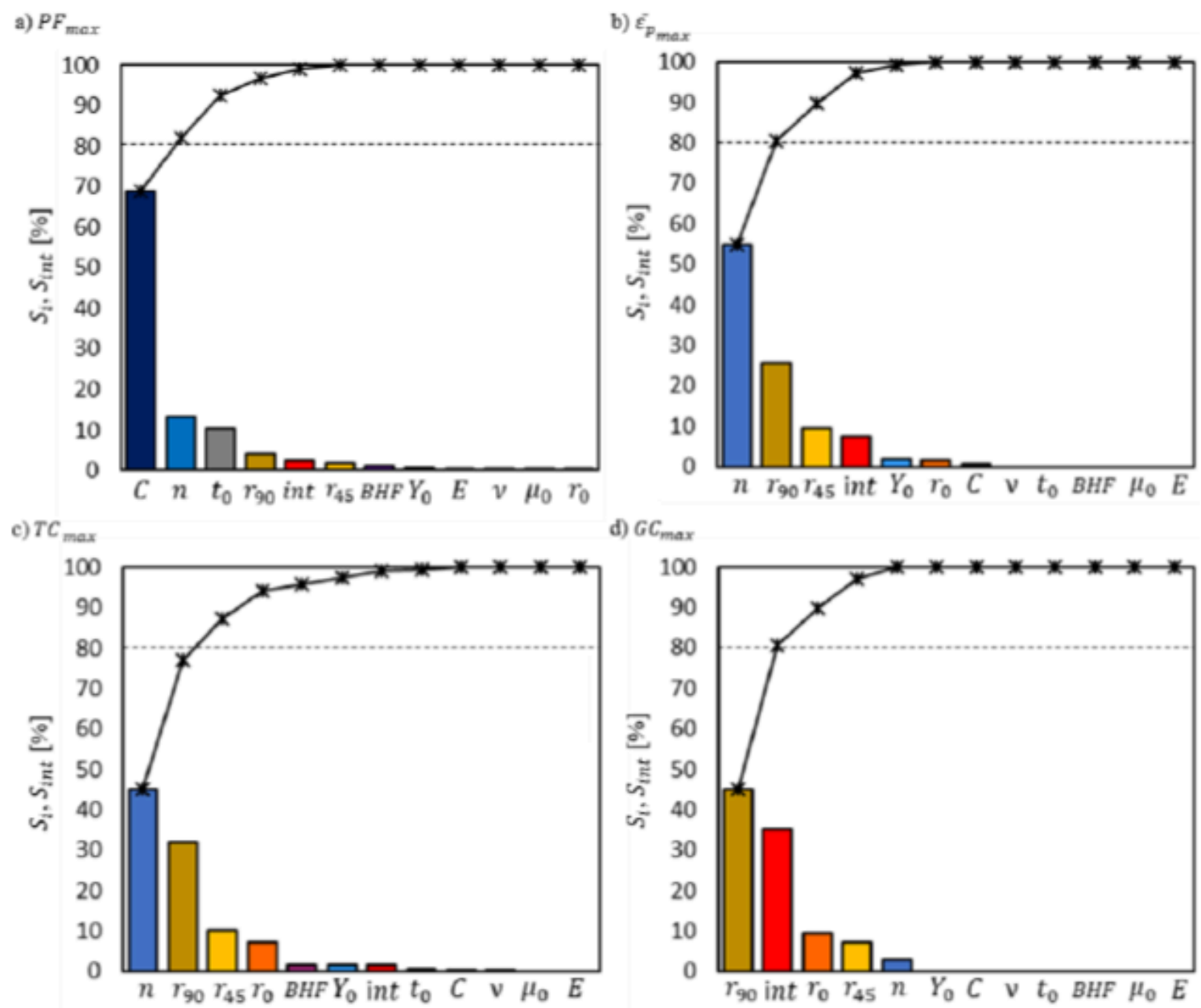

d) $G C_{\max }$

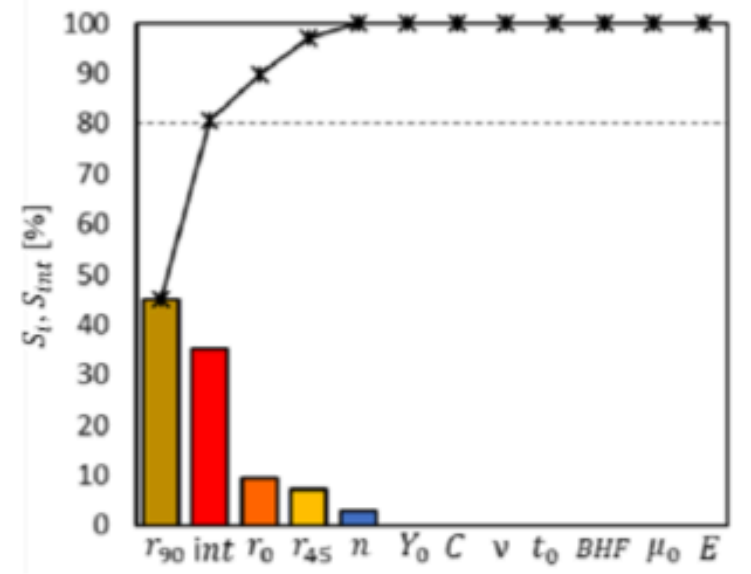

Fig. $4.1^{\text {st }}$ order indices of the input parameters, $S_{i}$, and sensitivity index of the interactions between input parameters, Sint, for the maximum values of the forming results:

a) $P F_{\max }$, b) $\bar{\varepsilon}^{p} \max$, c) $T C_{\max }$ and d) $G C_{\max }$. The black lines represent the cumulative value
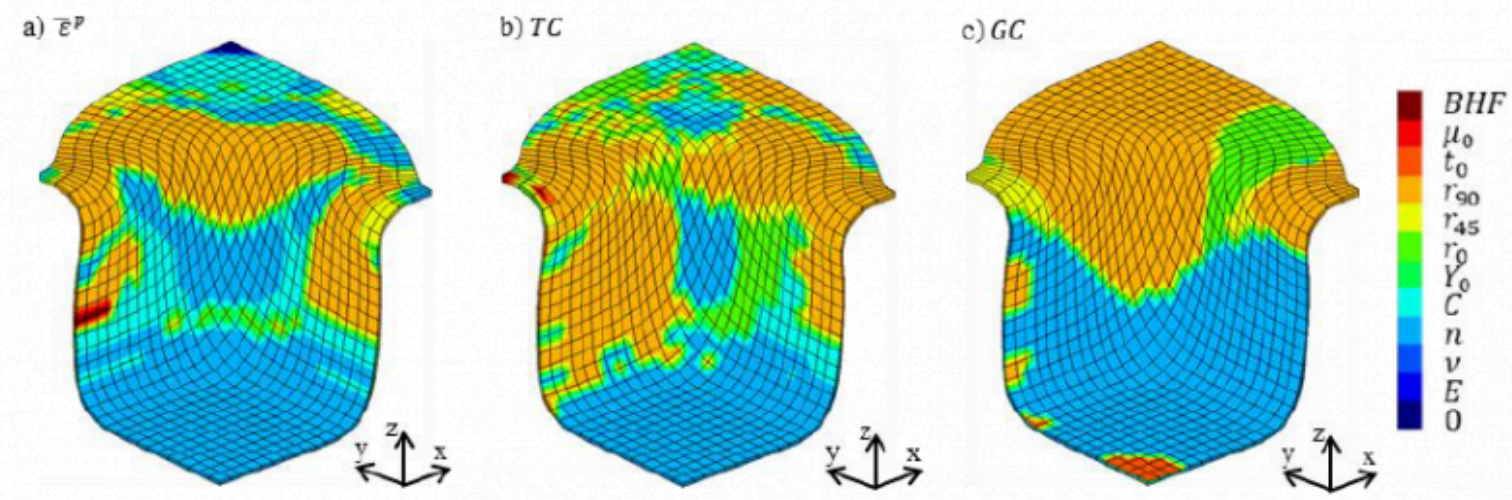

Fig. 5. Representation of the input parameter whose 1 st order index $S_{i}$ is maximum for the forming results: a) $\bar{\varepsilon}^{p}$, b) $T C$ and c) $G C$. 
Numerical study of the square cup stamping process: a stochastic analysis

The value " 0 " corresponds to a region of the cup that has no variability in the forming result $\bar{\varepsilon}^{p}$

\section{Conclusions}

In this work, a numerical study was performed to evaluate the influence of material and process uncertainty in the variability of the square cup forming results. For this purpose, uncertainty in the elasticity parameters, anisotropy coefficients, hardening parameters, blank thickness, friction coefficient and blank holder force was considered. The effect of these uncertainties is evaluated in the forming results of punch force, equivalent plastic strain, thickness change and geometry change.

A quasi-Monte Carlo method coupled with the Sobol's sequence was used to evaluate the mean and standard deviation values of the simulation results, by considering the uncertainty of the input parameters. With this analysis, it was possible to conclude that the geometry change is the result most sensitive to the uncertainty of the input parameters, with a maximum coefficient of variation equal to $56.75 \%$. Moreover, the flange and wall of the square cup are the regions where the effect of the input uncertainty is more significant. Afterwards, a variance-based sensitivity analysis (Sobol indices) was performed, in order to identify the input parameters with more effect in the forming results variability. It was concluded that the most influent inputs are the anisotropy coefficients and the hardening parameters, $n$ and $C$; the effect of the interactions between input parameters is only relevant for the geometry change.

The computation of the Sobol indices required a huge computational cost. With the goal of reducing this cost, metamodeling techniques will be used and their performance will be compared in future works. The sensitivity results of the square cup benchmark test will be used as reference.

\section{Acknowledgements}

This research is sponsored by FEDER funds through the program COMPETE - Programa Operacional Factores de Competitividade and by national funds through FCT - Fundação para a Ciência e a Tecnologia, under the project UIDB/ 00285/2020. It was also supported by projects: RDFORMING (reference PTDC/EME-EME/31243/2017), co-funded by Portuguese Foundation for Science and Technology, by FEDER, through the program Portugal-2020 (PT2020), and by POCI, with reference POCI-01-0145-FEDER-031243; EZ-SHEET (reference PTDC/EME-EME/31216/2017), co-funded by Portuguese Foundation for Science and Technology, by FEDER, through the program Portugal-2020 (PT2020), and by POCI, with reference POCI-01-0145-FEDER-031216. All supports are gratefully acknowledged.

\section{Bibliography}

[1] Col A (2003) Investigation on press forming scatter origin. In: Proceedings of the 6th international conference on material forming. pp 183-6

[2] Li YQ (2005) Application of Six Sigma Robust Optimization in Sheet Metal Forming. In: AIP Conference Proceedings. AIP, pp 819-824

[3] Arnst M, Ponthot J-P, Boman R (2018) Comparison of stochastic and interval methods for uncertainty quantification of metal forming processes. Comptes Rendus Mécanique 346:634-646. https://doi.org/10.1016/j.crme.2018.06.007

[4] Shahi VJ, Masoumi A, Franciosa P, Ceglarek D (2018) Quality-driven Optimization of Assembly Line Configuration for Multi-Station Assembly Systems with Compliant Non-ideal Sheet Metal Parts. Procedia CIRP 75:45-50. https://doi.org/ 10.1016/j.procir.2018.02.022 
[5] Dwivedy M, Kalluri V (2019) The effect of process parameters on forming forces in single point incremental forming. Procedia Manuf 29:120-128. https://doi.org/10.1016/j.promfg.2019.02.116

[6] Prates PA, Adaixo AS, Oliveira MC, Fernandes J V. (2018) Numerical study on the effect of mechanical properties variability in sheet metal forming processes. Int J Adv Manuf Technol 96:561-580. https://doi.org/10.1007/ s00170-018-1604-y

[7] Marques AE, Prates PA, Pereira AFG, et al (2020) Performance Comparison of Parametric and Non-Parametric Regression Models for Uncertainty Analysis of Sheet Metal Forming Processes. Metals (Basel) 10:457. https://doi.org/ $10.3390 /$ met10040457

[8] Huang C, Radi B, Hami A El (2016) Uncertainty analysis of deep drawing using surrogate model based probabilistic method. Int J Adv Manuf Technol 86:3229-3240. https://doi.org/10.1007/s00170-016-8436-4

[9] Fruth J, Roustant O, Kuhnt S (2019) Support indices: Measuring the effect of input variables over their supports. Reliab Eng Syst Saf 187:. https://doi.org/10.1016/j.ress.2018.07.026

[10] Zhu P, Zhang L, Zhou R, et al (2012) A novel sensitivity analysis method in structural performance of hydraulic press. Math Probl Eng 2012:. https://doi.org/10.1155/2012/647127

[11] Kim SH, Huh H (2002) Design sensitivity analysis of sheet metal forming processes with a direct differentiation method. In: Journal of Materials Processing Technology

[12] Sobol' I. (2001) Global sensitivity indices for nonlinear mathematical models and their Monte Carlo estimates. Math Comput Simul 55:271-280. https://doi.org/10.1016/S0378-4754(00)00270-6

[13] Bayraktar E, Altintaş S (1993) Square Cup Deep Drawing Experiments. In: NUMISHEET '93: Proceedings of the 2nd International Conference Numerical Simulation of 3-D Sheet Metal Forming Processes. Isehara, Japan, p 441

[14] Hama T, Takamura M, Makinouchi A, et al (2008) Effect of tool-modeling accuracy on square-cup deep-drawing simulation. Mater Trans 49:. https://doi.org/10.2320/matertrans.P-MRA2007885

[15] Li YQ, Cui ZS, Ruan XY, Zhang DJ (2006) CAE-Based six sigma robust optimization for deep- drawing process of sheet metal. Int J Adv Manuf Technol 30:. https://doi.org/10.1007/s00170-005-0121-y

[16] Padmanabhan R, Baptista AJ, Oliveira MC, Menezes LF (2007) Effect of anisotropy on the deep-drawing of mild steel and dual-phase steel tailor-welded blanks. J Mater Process Technol 184: https://doi.org/10.1016/ j.jmatprotec.2006.11.051

[17] Gutiérrez Regueras JM, Camacho López AM (2014) Investigations on the influence of blank thickness (t) and length/wide punch ratio (LD) in rectangular deep drawing of dual-phase steels. Comput Mater Sci 91:. https://doi.org/ 10.1016/j.commatsci.2014.04.024

[18] Neto DM, Oliveira MC, Menezes LF (2017) Surface Smoothing Procedures in Computational Contact Mechanics. Arch Comput Methods Eng 24:37-87. https://doi.org/10.1007/s11831-015-9159-7

[19] Menezes LF, Teodosiu C (2000) Three-dimensional numerical simulation of the deep-drawing process using solid finite elements. J Mater Process Technol 97:100-106. https://doi.org/10.1016/S0924-0136(99)00345-3

[20] Dib MA, Oliveira NJ, Marques AE, et al (2019) Single and ensemble classifiers for defect prediction in sheet metal forming under variability. Neural Comput Appl. https://doi.org/10.1007/s00521-019-04651-6 
Numerical study of the square cup stamping process: a stochastic analysis

[21] Prates PA, Marques AE, Oliveira MC, Fernandes J V. (2019) Comparing metamodeling techniques for variability analysis in sheet metal forming processes. In: AIP Conference Proceedings. p 120003

[22] Sobol' I. (1967) On the distribution of points in a cube and the approximate evaluation of integrals. USSR Comput Math Math Phys 7:86-112. https://doi.org/10.1016/0041-5553(67)90144-9

[23] Burhenne S, Jacob D, Henze G (2011) Sampling based on Sobol' sequences for Monte Carlo techniques applied to building simulations. In: Proceedings of Building Simulation. Sydney

[24] Kucherenko S, Albrecht D, Saltelli A (2015) Exploring multi-dimensional spaces: a Comparison of Latin Hypercube and Quasi Monte Carlo Sampling Techniques

[25] Saltelli A, Ratto M, Andres T, et al (2008) Global Sensitivity Analysis. The Primer

[26] Janon A, Klein T, Lagnoux A, et al (2014) Asymptotic normality and efficiency of two Sobol index estimators. ESAIM - Probab Stat 18:342-364. https://doi.org/10.1051/ps/2013040

PDF automatically generated on 2021-05-20 05:43:07

Article url: https://popups.uliege.be/esaform21/index.php?id=2158

published by ULiège Library in Open Access under the terms and conditions of the CC-BY License

(https://creativecommons.org/licenses/by/4.0) 